\title{
Pattern goodness and redundancy revisited: Multidimensional scaling and hierarchical clustering analyses
}

\author{
ROBERT J. GLUSHKO \\ Stanford University, Stanford, California 94305
}

\begin{abstract}
Twenty subjects made all possible (136) pairwise-comparison judgments of pattern goodness between 17 five-dot patterns. Using multidimensional scaling and hierarchical clustering analyses, a two-dimensional representation was judged the most appropriate for the derived pattern space. The interpreted structure of the spatial-hierarchical configuration strongly supported previous findings that pattern redundancy is the most salient dimension underlying pattern goodness judgments and that patterns can be partitioned on this dimension according to the size of their equivalence sets, i.e., the number of patterns resulting from rigid rotations in 90-deg increments or reflection. A psychological mechanism that arcounts for the effect of pattern redundancy on judged goodness was proposed.
\end{abstract}

Some patterns are perceived as better than others. The Gestalt psychologists emphasized principles of good form, but it remained for Attneave (1954) to notice that the Gestalt notions of figural organization pertained essentially to information distribution. A gc...: Gestalt is a figure with a high degree of structural redundancy; the laws of symmetry, closure, continuation, common fate, and proximity are all related to conditions reducing perceptual uncertainty. Hochberg and McAlister (1953) found that figural organizations requiring less information to define were more easily perceived, and hence better patterns, than less redundant organizations.

Garner (1962) pointed out the inconsistency in using the concept of redundancy with respect to single patterns. He proposed that redundancy is more properly seen as a property shared by a set of patterns, and that a pattern's goodness is related to its redundancy as measured by the number of patterns inferred from or equivalent to it. When a pattern is perceived. it suggests a set of alternatives; the more redund ant the pattern, the fewer different alternatives it implies. The very best patterns are so "good" that they suggest only themselves, and thus have an inferred "equivalence set" of size one.

To test the hypothesis that pattern goodness is inversely related to the equivalence set size, Garner and Clement (1963) had subjects rate the goodness of 90 patterns on a scale of $1-7$ in decreasing goodness and then sort the patterns into groups of similar goodness. They obtained a correlation of .84 between goodness ratings and the size of the group that each

The author is indebted to Roger Shepard and Robert Sternberg for critical readings of an earlier version of this paper. The author is now at the Department of Psychology, University of California at San Diego, La Jolla, California 92037. pattern was sorted into, supporting their theory that redundancy, as defined in terms of the size of the equivalence set, is fundamental to goodness. Good patterns are perceived as being more unique and as belonging to small psychologically inferred sets, while poorer patterns suggest large sets of alternative figures. Garner and Clement proposed that an objective measure of the equivalence set size for each pattern was the number of perceptually different patterns resulting from rigid rotation in $90-$ deg steps or rellection. The equivalence set defined this way accounted for $73 \%$ of the total variance of the pattern goodness ratings.

Steinheiser (1970) had subjects make the 120 possible pair-comparison similarity ratings of 16 abstract patterns and then make a preference for one member of each pair. The interpattern distances in a multidimensional scaling configuration derived from the pooled similarity data reflected the pattern preferences of the individual subjects. As patterns were less preferred, their Euclidean distances from a subject's most preferred pattern increased. This result was obtained even though the most preferred pattern varied greatly between subjects. Similarity ratings adequately determined the psychological pattern preference space, even though subjects made their aesthetic judgments from different vantage points within the space. Nevertheless, to demonstrate that the derived pattern space has more than statistical relevance to pattern preferences, or to interpret MDS dimensions as reflecting pattern properties or judgmental criteria, one must compute the scaling solution from the preferences themselves rather than from similarity data. Similarity and preference data arise from different kinds of judgments and are not strictly interchangeable.

The present experiment is intended to test the 
pattern goodness and redundancy hypothesis as advanced by Attneave, Garner, and others while using the method of pairwise-preference goodness judgments and techniques of multidimensional scaling and hierarchical clustering instead of more traditional data analyses. Beyond the basic assumption that the psychological pattern space can be represented as a Euclidean configuration, the scaling algorithm introduces no other theoretical preconceptions about the structure of pattern goodness judgments, yet will reveal any relationships between the objective pattern properties and goodness preferences. Specitically, these methods of analysis will investigate the viability of Garner's (1962) suggestions that the dimension of redundancy is primary in pattern goodness and that equivalence sets can be viewed as partitions on this redundancy dimension (Garner, 1966).

\section{METHOD}

Subjects

Subjects were 20 Stanford University undergraduates who volunteered for the experiment.

\section{Stimuli}

The stimuli were the 17 patterns selected by Garner and Clement (1963) as typical of the set of 90 that can be constructed by placing five dots in the cells of an imaginary $3 \times 3$ square matrix with the requirement that each row and column contain at least one dot. Many of the patterns are equivalent in the sense that they are rigid rotations in 90-deg increments or reflections of one another. There are 17 such equivalence sets: 2 unique patterns (unchanged after rotation or reflection) that constitute equivalence sets of size one, 8 equivalence sets of size four, and 7 equivalence sets containing eight distinct patterns. Thus these 17 patterns in the present experiment, one from each equivalence set, represent the goodness range of the entire set of 90 patterns. The 17 patterns are shown in Figure 1.

The 1.36 different pair combinations of patterns were drawn on the backs of computer keypunch cards. The card was divided in half by a black line, and on each side was drawn one pattern of the pair. The dots were $1 / 8$-in. black circles and were centered on the cells of an imaginary $3 \times 3$ square matrix $2 \mathrm{in.}$ on a side.

Fach pattern was numbered in the lower left corner of each side of the test card to identify the patterns being judged. Each of the cards contained a number between 1 and 136 in the upper right corner. The patterns were placed on the right and left sides of the cards with equal frequency (8) to counter any possible bias for either side.

\section{Procedure}

Each subject was given the shuffled deck of 136 cards and an answer sheet. He was instructed to look at each card. decide which of the two patterns was "better," and then circle the appropriate pattern number for that card on the answer sheet. Subjects apparently had no difficulty with the undefined concept of goodness and the average subject completed the entire task in less than 30 min.

\section{RESULTS AND DISCUSSION}

The aggregate pairwise preferences from the 20 subjects were tabulated as a $17 \times 17$ lower-half matrix with the diagonal absent. With purely dichotomous

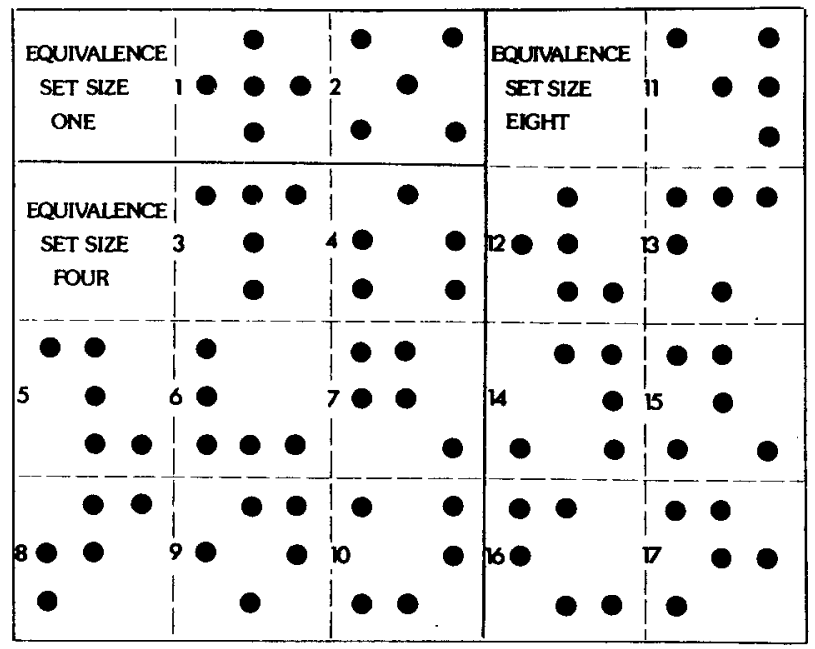

Figure 1. The 17 patterns used in the experiment. The size of the equivalence set for each pattern is the number of different visual configurations resulting from rigid rotation in 90-deg increments or reflection.

goodness preference data, a derived measure of pattern goodness is the departure from chance of a particular pattern's choice over another. Since dissimilar goodness between two patterns is implied by frequent choice of either one over the other, the absolute value of the difference between the observed and the expected frequency of a goodness preference represents the similarity of the pattern goodness of the two patterns in the 136 pairs. Small values indicate that the two patterns are of similar rated goodness, while large values indicate that judgments of dissimilar goodness were made. This transform of the original data yielded the input matrix for analysis by nonmetric multidimensional scaling of the type introduced by Shepard $(1962 \mathrm{a}, \mathrm{b})$ and Kruskal (1964). Specifically, Kruskal's program M-D-SCAL $5 \mathrm{M}$ was used. The original preference data and the transformed input matrix are shown in Table 1.

Ten multidimensional scaling solutions were obtained for one through four dimensions. The two-dimensional solution with the lowest stress (.0935) was judged the most appropriate representation of the psychological pattern space. Although no one criterion of goodness of fit was conclusive, an overall consideration of stress, reliability, and interpretability pointed to the particular configuration selected.

Stress Form 1, a "badness-of-fit" measure developed by Kruskal (1964) for MDS configurations, decreased regularly with more dimensions without the abrupt drop which might suggest that the inherent dimensionality of the space had been achieved. The slight "elbow" in the stress-vs.-dimensions graph 
Table 1

(a) Goodness Preferences*

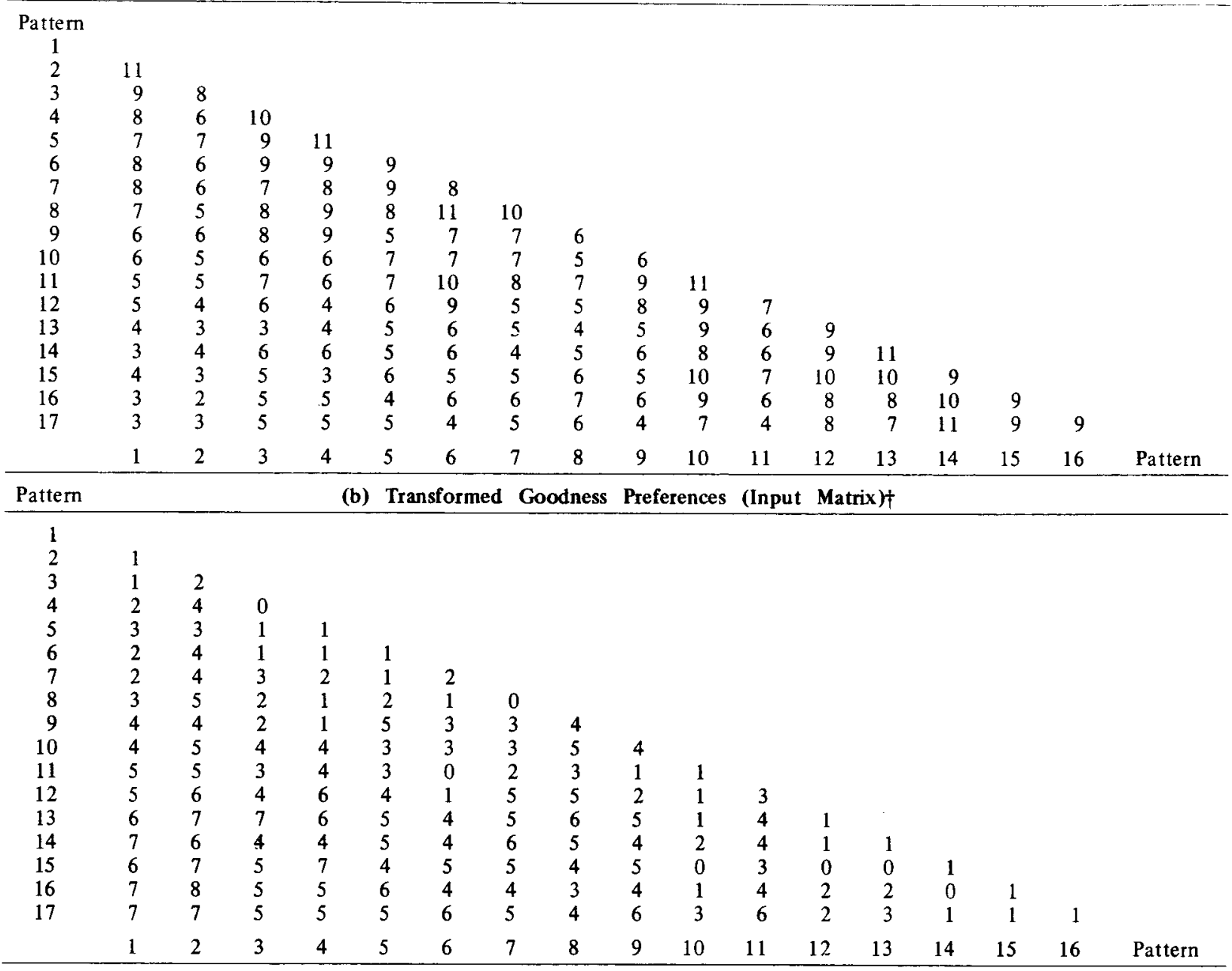

*Each entry is the number of times the pattern on the left was chosen as better than the pattern on the bottom.

tEach entry is a transform of the corresponding goodness preference value, the absolute value of the difference between the observed and expected preference frequency (since $n=20, e=10$ ).

(Figure 2) does not justify by itself the choice of the two-dimensional solution. The generally underestimated possibility that the MDS configuration with the lowest stress might represent a local minimum rather than the absolute best fit for that dimensionality and the desire for an interpretable solution in two or at most three dimensions override any substantial reliance on elbows or stress alone.

Johnson's (1967) HICLUS algorithm for hierarchical clustering was also applied to the derived data matrix. Although clustering solutions have no inherent spatial representation, Shepard (1972) proposed that embedding a hierarchical clustering solution in a MDS spatial representation can provide a check on the adequacy of the spatial configuration. Very elongated or convoluted clusters suggest that the scaling representation may be in too few dimensions to avoid excessive stress or that the particular solution corresponds to a local minimum. That the hierarchical solution fits as well as Figure 3 indicates enhances the credibility of the scaling representation.

The most compelling reason for accepting the two-dimensional solution is its interpretability. The primary dimension in the space is the redundancy dimension as measured by Garner and Clement's (1963) notion of equivalence set size for each of the 17 patterns. The largest clusters obtained from the hicrarchical clustering diameter method recover the equivalence sets in their proper order along the redundancy dimension. That redundancy is the dominant correlate of pattern goodness is demonstrated by the rank-order correlation of .97 between overall pattern preference (the total number of times each pattern was chosen as the better pattern 


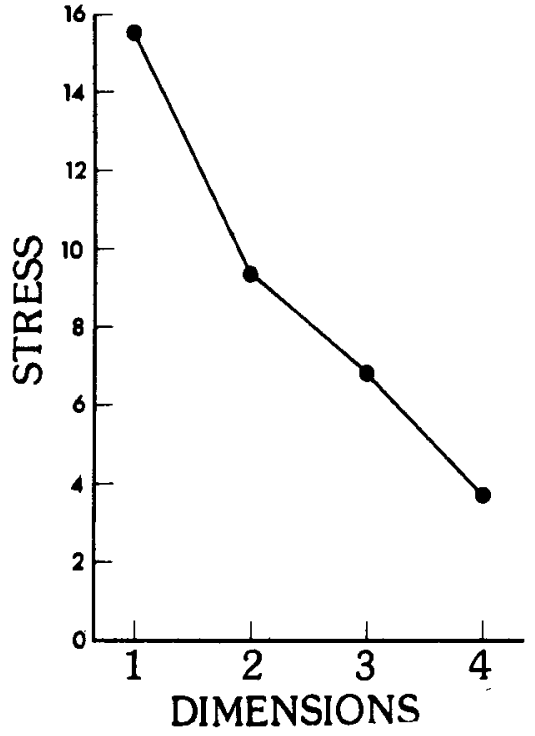

Figure 2. Relation of atress (percent) to the number of dimensions in the multidimensional scalling colution. The values are the lowest obtained for each dimenolonality in 10 run begun with random starting configurations.

of a pair) and the order of projection of the patterns on the best-fitting redundancy axis in the MDS configuration.

The second dimension in the pattern space is not as well defined as the redundancy axis, but appears to reflect variations in the overall area and perimeter of. the patterns. Patterns that occupy more of the inaginary matrix were generally preferred over more compact patterns with the same degree of redundancy. The correlation of overall pattern preference with the projections on the best-fitting dispersion-compactness axis is .74 , suggesting that this dimension is not as important in pattern good ness as is redundancy. Perhaps this dimension is salient only in discriminations between patterns of similar redundancy.

The third and fourth dimensions in higher dimensional solutions were not reliably interpretable. The nature of multidimensional scaling makes it difficult to assess the significance of straight lines and other stimulus properties highly correlated with redundancy.

In general, the structure of the pattern space as determined through multidimensional scaling and hierarchical clustering techniques is interpretable and validating down to rather fine distinctions. The rank-order correlation between the overall preference order for the 17 patterns used in the present experiment and Garner and Clement's (1963) goodness ratings for the same patterns is .91. The clustering solution partitions the equivalence sets noted by Garner and Clement, those with one, four, or eight alternatives upon rotation or reflection, with only two exceptions. Pattern 10 (see Figure 1), with an equivalence set of size four, clusters with the eight-equivalent patterns, while Pattern 11, with an equivalence set of eight, is recovered in the four-equivalent cluster. Garner and Clement reported this same reversal in goodness ratings for these two patterns; thus, the only departure from prediction on the basis of equivalence set size is, in fact, predictable on the basis of their data.

Steinheiser's (1970) results suggested the possibility that a composite pattern space might accurately reflect pattern preferences, even though the dimensions of preference varied between subjects. Since he used abstract patterns and an undirected criterion of preference, that finding was not alarming, but in an experiment designed to investigate pattern goodness preference rather than simply pattern preference, it is imperative that patterns in the "good" regions of the MDS configuration actually be those most preferred by individual subjects and not just those preferred in the pooled data. Nevertheless,

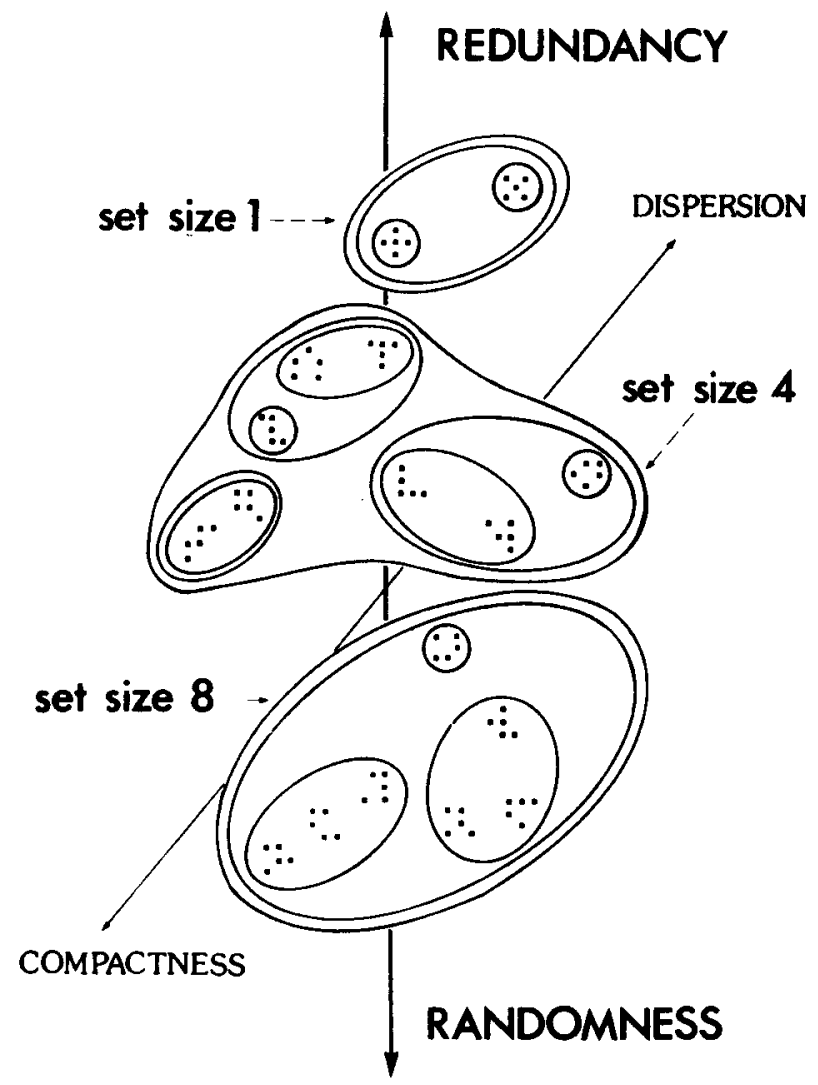

Figure 3. "Cuts" from the hierarchical clustertng solution embedded in the lowest stress two-dimensional multidimensional scaling configuration. The largest clusters recover the equivalence sets of size one, four, and eight. 
this possibility is discounted by the fact that the most preferred pattern for 13 of ther 20 subjects was one of the two unique ones (with equivalence set size one) and the other 7 subjects most preferred one of the better patterns of equivalence set size four.

It has been demonstrated that redundancy has explanatory value as a dimension of pattern good ness judgments. but the substantive issue of what prichological mechanism accounts for the strong clfect of equivalence set size on pattern goodness rumains unanswered. Garner (1974) saw goodness more as a property inherent in certain patterns than as a result imparted by the perceiver. He noted that "our experimental problem in this case is to investigate the properties of stimuli that lead to different inferred sets of stimuli ... some stimuli have properties which lead to inferred subsets with little need for, or effect of, prior experience [p. 11]. "Yet in the notion of "inferred subset" is implicit the acknowledgment that the perceiver somehow carries out, or at least recognizes the consequences of varying out, the transformations of rotation and reflection that give rise to the equivalence set for each pattern. Rock (1973) suggested a more cognitive theory of form perception that required taking into account external as well as internal figural properties, and in particular, required the implicit or unconscious assignment of direction to the figure on the basis of perceptual cues. These cues not only consist of those noted by Garner that exist in the pattern structure, but also include environmental features, such as the visual reference frame and gravity, and cognitive elements, such as the perceiver's egocentric coordinates and instructional set. Rock argued that "phenomenal shape is affected by such assignment of directions because the implicit cognitive description is a function of the figure's directions [p. 126]." The eye does not simply respond to stimulus redundancy or symmetry, but must actively seek it out in order to perceive the nature of the form.

Perhaps pattern goodness can be explained in terms of the ease with which the perceiver can implicitly assign directions to the pattern. The best patterns appear identical in all of the eight rotated or reflected positions because they are symmetric on both axes. Thus, the process of assigning directions, guided as it is by the symmetry and intrinsic direction of a form, is easy regardless of the orientation of the pattern. Poor patterns, on the other hand. have large equivalence sets because the structure of the pattern does not produce the same visual configuration upon rotation or reflection, and in some orientations the assignment of directions to the figure is difficult. Some amount of trial and error or exploratory rotation and reflection might be necessary before the assignment of directions can occur when the perceptual cues are ambiguous or less well defined. This explanation is speculative but is consistent with the evidence that good tigures are those with unequivocal percepts and poor figures are those producing perceptual uncertainty. Good patterns, then, may result when the structural properties of figures allow the perceiver to assign directions more easily and thus more easily to perceive.

\section{REFERENCES}

Atrneave, F. Some informational aspects of visual perception. Psychological Review, 1954, 61, 183-193.

GARNER, W. R. Uncertainty and structure as psychological concepts. New York: Wiley, 1962.

Garner, W. R. To perceive is to know. American Psychologist, $1966,21,11-19$.

GARNER, W. R. The processing of information and structure. Potomac, Md: Lawrence Erlbaum, 1974.

Garner, W. R., \& Clement, D. E. Goodness of pattern and pattern uncertainty. Journal of Verbal Learning and Verbal Behavior, 1963, 2, 446-452.

HocheerG, J., \& MCAlister, E. A quantitative approach to tigural goodness. Journal of Experimental Psychology. 1953, 46, 361-364.

Johnson, S. C. Hierarchical clustering schemes. Psychometrika. 1967, 32. 241-254.

Kruskal, J. B. Multidimensional scaling by optimizing goodness of tit to a nonmetric hypothesis. Psychometrika, 1964, 29, 1-28.

Rock, I. Orientation and form. New York: Academic Press, 1973.

SHEPARD, R. N. The analysis of proximities: Multidimensional scaling with an unknown distance function. I. Psychometrika, 1962, 27. 135.140. (a)

Shepard, R. N. The analysis of proximities: Multidimensional scaling with an unknown distance function. II. Psychometrika, 1962, 27, 219-246. (b)

SHEPARD, R. N. Psychological representation of speech sounds. In E. E. David and P. B. Denes (Eds.), Human communication: A unified view. New York: McGraw-Hill, 1972.

Steinheiser, F. H., JR. Individual preference scales within a multidimensional "similarities" space. Journal of Experimental Psychology, 1970, 86, 325-327.

(Received for publication August 21, 1974; accepted September 26, 1974.) 